\title{
Relação entre resiliência e depressão em pacientes com câncer de cabeça e pescoço
}

\author{
Relationship between resilience and depression in patients with head and neck cancer \\ Relación entre resiliencia y depresión en pacientes con cáncer de cabeza y cuello
}

Recebido: 07/07/2021 | Revisado: 09/07/2021 | Aceito: 10/07/2021 | Publicado: 21/07/2021

Ywia Danieli Valadares

ORCID: https://orcid.org/0000-0003-4124-6309

Universidade Federal de São João del Rei, Brasil

E-mail: ywiaval@ hotmail.com

João Marcos Arantes Soares

ORCID: https://orcid.org/0000-0002-9735-3026

Universidade Federal de São João del Rei, Brasil

E-mail: jmarcosccp@uol.com.br

Ana Paula Madureira

ORCID: https://orcid.org/0000-0002-6614-7940

Universidade Federal de São João del Rei, Brasil

E-mail: apmadureira@ufsj.edu.br

Ydia Mariele Valadares

ORCID: https://orcid.org/0000-0001-6028-551X

Universidade Federal de Juiz de Fora, Brasil

E-mail: ydia.valadares@ufjf.edu.br

Michele Conceição Pereira

ORCID: https://orcid.org/0000-0001-8866-7499

Universidade Federal de São João del Rei, Brasil

E-mail: michelepereira@ufsj.edu.br

\begin{abstract}
Resumo
Objetivo: Comparar os escores de resiliência e depressão em pacientes com carcinoma de células escamosas de cabeça e pescoço (CCECP) submetidos a tratamento cirúrgico em diferentes momentos do tratamento e correlacionar tais variáveis. Metodologia: Trata-se de um estudo de coorte, desenvolvido com 28 pacientes diagnosticados com CCECP, submetidos a tratamento cirúrgico e acompanhados no pré-operatório (T1), quatro a seis semanas (T2) e seis meses após o término do tratamento (T3). Aplicaram-se o Mini exame do estado mental (MEEM), a Escala de resiliência e o Inventário de depressão de Beck nos três momentos. Resultados: Comparando-se a resiliência, seus domínios e a depressão nos três tempos, não houve diferença significativa. Notou-se correlação positiva entre a resiliência e a depressão. Conclusão: Perante a relação entre resiliência e depressão observada, sugere-se que essas variáveis sejam avaliadas nesse grupo de pacientes, a fim de ajudar a equipe a inferir quais deles podem se beneficiar de uma intervenção precoce, a trabalhar estratégias para reduzir os níveis de depressão e aumentar a resiliência, contribuindo para o sucesso do tratamento.
\end{abstract}

Palavras-chave: Neoplasias de cabeça e pescoço; Carcinoma de células escamosas; Resiliência psicológica; Depressão.

\begin{abstract}
Objective: To compare the resilience and depression scores in patients with head and neck squamous cell carcinoma (HNSCC) undergoing surgical treatment at different times of treatment and to correlate these variables. Methodology: This is a cohort study, developed with 28 patients diagnosed with HNSCC, undergoing surgical treatment and followed up preoperatively (T1), four to six weeks (T2) and six months after the end of treatment (T3). The Mini Mental State Examination (MMSE), the Resilience Scale and the Beck Depression Inventory were applied in three moments. Results: Comparing resilience, its domains and depression in the three stages, there was no significant difference. A positive correlation was noted between resilience and depression. Conclusion: Given the relationship between resilience and depression observed, it is suggested that these variables be evaluated in this group of patients, to help the team to infer which of them can benefit from an early intervention, to work on strategies to reduce levels of depression and increase resilience, contributing to treatment success.
\end{abstract}

Keywords: Head and neck neoplasms; Squamous cell carcinoma; Psychological resilience; Depression.

\section{Resumen}

Objetivo: Comparar las puntuaciones de resiliencia y depresión en pacientes con carcinoma de células escamosas de cabeza y cuello (CCECC) sometidos a tratamiento quirúrgico en diferentes momentos de tratamiento y correlacionar estas variables. Metodología: Se trata de un estudio de cohorte, desarrollado con 28 pacientes diagnosticados de CCECC, sometidos a tratamiento quirúrgico y seguidos preoperatoriamente (T1), cuatro a seis semanas (T2) y seis 
meses después de finalizado el tratamiento (T3). El Mini Examen del Estado Mental (MMSE), la Escala de Resiliencia y el Inventario de Depresión de Beck se aplicaron en tres momentos. Resultados: Al comparar la resiliencia, sus dominios y la depresión en las tres etapas, no hubo diferencia significativa. Se observó una correlación positiva entre resiliencia y depresión. Conclusión: Dada la relación entre resiliencia y depresión observada, se sugiere evaluar estas variables en este grupo de pacientes, con el fin de ayudar al equipo a inferir cuáles de ellos pueden beneficiarse de la intervención temprana, para trabajar en estrategias para reducir los niveles de depresión y aumentar la resiliencia, contribuyendo al éxito del tratamiento.

Palabras clave: Neoplasias de cabeza y cuello, Carcinoma de células escamosas, Resiliencia psicológica, Depresión.

\section{Introdução}

Câncer de cabeça e pescoço (CCP) se refere aos tumores localizados na cavidade oral, lábios, faringe e laringe (GomesFerraz et al., 2019; Algtewi et al.,2017). Com grande impacto mundial, correspondem a aproximadamente 744.000 novos casos diagnosticados no último ano, sendo quase metade deles no lábio e cavidade oral (Sung et al., 2021). No Brasil, 22.840 novos casos serão diagnosticados no triênio 2020-2022, sendo a maior incidência na cavidade oral (15.210 novos casos), ocupando a quinta posição (INCA, 2019).

Grande parte dos tumores de cabeça e pescoço $(\mathrm{CP})$ atinge as células escamosas que revestem as mucosas do trato digestivo superior, sendo 90\% dos casos classificados como carcinomas de células escamosas (CCE) (Siegel et al., 2020).

As opções de tratamento são baseadas na localização do tumor, estadiamento clínico, condições clínicas e físicas do paciente, além da ressecabilidade do tumor e manutenção dos órgãos (Huang et al., 2019). Para as neoplasias em estágios iniciais, opta-se por cirurgia ou radioterapia e para os estágios mais avançados, faz-se uma associação de cirurgia e radioterapia ou quimioterapia (Rocha et al., 2017). Essas intervenções terapêuticas, isoladas ou associadas, geralmente têm efeitos colaterais agudos que comprometem a funcionalidade, tais como: distúrbios na fala, deglutição, respiração, paladar, olfato e desfiguração facial (Ahn et al., 2015; Wu et al., 2016), as quais podem causar graves consequências a longo prazo e até óbito (Talani et al., 2019).

Estudos demonstram que o sofrimento psicológico diante da descoberta da doença e o avanço do tratamento acompanham esses pacientes, incluindo sintomas de depressão (Krebber et al., 2016; Paula et al., 2012). Pacientes com CCECP, assim como outros pacientes oncológicos, tem probabilidade aumentada para o desenvolvimento de transtornos depressivos, visto que se deparam com o risco de vida e sobretudo, a morbidade acarretada pelo tratamento (Kim et al., 2016).

A relação entre a depressão, prognóstico da doença e mortalidade tem sido estudada. Em pacientes deprimidos a chance de morrer é 26 a 39\% maior que naqueles não deprimidos (Rieke et al., 2017; Satin et al., 2009; Zimmaro et al., 2018). A redução da sobrevida de pacientes com CCP e depressão pode estar associada à desnutrição decorrente do transtorno psicológico, que contribui para redução da imunidade e aumento da morbimortalidade (Britton et al., 2012). Edmonds \& Mcguire (2007) observaram que pacientes com CCP e deprimidos tendem a ter pequena aderência ao tratamento (quimioterapia e/ou radioterapia), contribuindo para pior prognóstico e redução da sobrevida.

Diante de toda dor causada pela doença e seu tratamento, alguns pacientes tornam-se mais resilientes e suplantam as dificuldades e utilizam-se delas como uma forma de desenvolvimento individual (Lima et al., 2020). Estudos sobre a resiliência aumentaram nos últimos anos e sua investigação em pacientes oncológicos tem crescido (Aguiar, Meira \& Raquel, 2011; Fernández \& García, 2012).

Em pacientes com CCP ainda existe uma escassez de trabalhos envolvendo a resiliência, até o momento apenas dois estudos foram encontrados relacionados a essa temática. Clarke et al., (2019) estudaram resiliência e qualidade de vida (QV) nesse grupo, por meio de um estudo retrospectivo, sem acompanhamento dos pacientes e que não levou em consideração a depressão. Valadares et al., (2021) avaliaram resiliência, QV e depressão em pacientes com CCP, porém somente no período 
pré-operatório. Sendo assim, esse estudo é pioneiro na investigação da relação entre resiliência e depressão nessa população após tratamento cirúrgico.

\section{Metodologia}

Trata-se de um estudo clínico longitudinal prospectivo, de coorte, com abordagem quantitativa, desenvolvido com pacientes diagnosticados com CCECP e submetidos a tratamento cirúrgico no Departamento de Cirurgia de Cabeça e Pescoço de um hospital de referência no interior de Minas Gerais, Brasil, no período de agosto de 2017 a novembro de 2019.

Essa pesquisa foi aprovada pelo Comitê de Ética em Pesquisa Envolvendo Seres Humanos do Campus Centro-Oeste Dona Lindu, da Universidade Federal de São João Del Rei (protocolo no 2.172.356) e pelo Comitê de Ética em Pesquisa do Hospital São João de Deus (protocolo n ${ }^{\circ}$ 2.225.907). Todos os voluntários assinaram o termo de consentimento livre e esclarecido (TCLE).

Para avaliar a resiliência e a depressão, os questionários foram aplicados em três momentos distintos: pré-operatório (Tempo 1- T1); entre quatro a seis semanas (Tempo 2- T2) e seis meses após o término do tratamento (Tempo 3- T3). Os critérios de inclusão compreenderam: (I) pacientes com tumor primário diagnosticado como CCE localizados na boca, faringe, laringe ou hipofaringe, confirmado por biópsia; (II) pacientes submetidos à cirurgia como tratamento inicial com finalidade curativa; (III) ausência de alterações cognitivas identificadas pelo Mini Exame do Estado Mental (MEEM) (Bertolucci et al., 1994); (IV) pacientes que concordaram com o estudo e assinaram o TCLE. Os critérios de exclusão foram: (I) presença de outros tumores primários simultâneos; (II) pacientes com metástase à distância na época da admissão e (III) ausência de realização de alguma das avaliações do protocolo.

Após a avaliação dos cirurgiões e indicação do procedimento cirúrgico, realizou-se a coleta de dados do T1. Cada voluntário respondeu a uma ficha de identificação contendo dados demográficos, história clínica, fatores de risco e exame locorregional.

Aplicou-se o MEEM para avaliação cognitiva, composto por sete categorias que mensuram funções cognitivas específicas: orientação para tempo e local, registro de três palavras, atenção e cálculo, lembrança de três palavras, linguagem e capacidade construtiva visual (Bertolucci et al., 1994). O escore varia de zero a 30 pontos, sendo: 20 pontos para analfabetos, 25 pontos para idosos com um a quatro anos de estudo, 26,5 pontos para idosos com cinco a oito anos de estudo, 28 pontos para aqueles com nove a 11 anos de estudo e 29 pontos para aqueles com mais de 11 anos de estudo (BRUCKI et al., 2003).

Adicionalmente, os pacientes responderam ao Inventário de depressão de Beck, composto por 21 questões objetivas, variando de zero a três pontos. Os itens descrevem a tristeza, pessimismo, sensação de fracasso, falta de satisfação, sensação de culpa, sensação de punição, autodepreciação, autoacusações, ideias suicidas, crises de choro, irritabilidade, retração social, indecisão, distorção da imagem corporal, inibição para o trabalho, distúrbio do sono, fadiga, perda de apetite, perda de peso, preocupação somática e diminuição de libido, numa pontuação de 0 a 63. Escores de 0 a 11: indicam pacientes sem depressão ou depressão mínima; escores de 12 a 19: depressão leve; escores de 20 a 35: depressão moderada e escores de 36 a 63: depressão grave (Gorestein \& Andrade, 1998).

Por fim, os participantes responderam a escala de resiliência desenvolvida por Wagnild e Young, (1993), a qual avalia os níveis de adaptação psicossocial positiva em face de eventos de vida importantes e divide-se em três domínios: resolução de ações e valores, independência e determinação e autoconfiança e capacidade de adaptação a situações. Validada no Brasil por Pesce et al., (2005), esse instrumento possui 25 itens descritos de forma assertiva, cujas respostas especificam o nível de concordância do entrevistado à afirmação (tipo likert), variando de um (discordo totalmente) a sete (concordo totalmente). Os escores da escala variam de 25 a 175 pontos, sendo que valores altos indicam elevada resiliência. A soma dos pontos gera um 
escore total que foi categorizado em: baixo (menor ou igual a 124 pontos); médio (125 a 145 pontos) e alto (maior que145 pontos) (Wagnild, 2009).

O inventário de depressão de Beck e a escala de resiliência foram aplicados também no T2 e T3, no momento em que cada paciente fazia o retorno ao hospital.

Os dados foram analisados pelo software Statistic Package for Social Sciences (SPSS) for Windows, versão 23.0 (SPSS Inc., Estados Unidos). Para verificar a diferença entre os três tempos avaliados, testou-se a normalidade dos dados por meio de teste de Kolmogorov-Smirnov. Como nenhuma das variáveis apresentou distribuição normal, realizou-se a análise de variância de Friedman (para medidas repetidas) seguida de pós teste de Dunn.

Na correlação entre as variáveis, o teste de Kolmogorov Smirnov não mostrou distribuição normal. Dessa forma, utilizou-se o teste de correlação de Spearman. O nível de significância de $5 \%(p \leq 0,05)$ foi considerado para todos os testes estatísticos.

\section{Resultados e Discussão}

No T1, avaliaram-se 34 pacientes com CCECP, porém seis foram excluídos por não terem cognitivo preservado, segundo o MEEM. Dos 28 pacientes do T1, seis foram excluídos: dois por decisão de tratamento conservador, um por se recusar a continuar na pesquisa e três por óbito, totalizando 22 pacientes. No T2 e na etapa final (T3), um paciente foi excluído, pois mudou-se de estado e perdemos o seguimento, contabilizando 21 pacientes.

$\mathrm{Na}$ análise da resiliência, observou-se que não houve diferença significativa em qualquer domínio nem no escore total nos três tempos investigados, como ilustra a Tabela 1.

Tabela 1. Média, mediana e intervalo da pontuação dos domínios de resiliência e escore total e comparação destes nos três tempos avaliados.

\begin{tabular}{ccccc}
\hline & $\begin{array}{c}\text { T1 } \\
\text { Média } \\
\text { Mediana } \\
\text { (Intervalo) }\end{array}$ & $\begin{array}{c}\text { T2 } \\
\text { Média } \\
\text { Mediana } \\
\text { (Intervalo) }\end{array}$ & $\begin{array}{c}\text { T3 } \\
\text { Média } \\
\text { Mediana } \\
\text { (Intervalo) }\end{array}$ & p \\
\hline Resolução de Ações e & 84 & 78,55 & 82,19 & \\
Valores & 88,5 & 84 & 84 & 0,9876 \\
Independência e & $57-98$ & $30-97$ & $35-98$ & \\
Determinação & 30,14 & 28,09 & 29,57 & 0,917 \\
Autoconfiança e & 30 & 28 & 30 & 0,318 \\
Capacidade de adaptação a & $18-42$ & $10-42$ & $13-42$ & \\
situações & 30,71 & 28,36 & 30,52 & \\
\hline & 33 & 29 & $18-35$ & 0,717 \\
Escore total & $16-59$ & $16-35$ & 142,29 & 149 \\
& 144,85 & 135,00 & $77-175$ & \\
\hline
\end{tabular}

*p valor $<0,005$ é considerado significante.

Fonte: Autores.

A ausência de diferença nos três tempos avaliados pode estar associada à moderada resiliência que os pacientes apresentavam no pré-operatório (média de 144,85), sugerindo que eles não passaram pela fase de negação e avançaram para o período da aceitação, na qual reconhecem que a doença, tratamento e evolução são inevitáveis, conseguem manter conversas 
mais duradouras sobre a doença, olham a vida nos aspectos positivos e negativos e demonstram maior maturidade (Cardoso et al., 2009), com isso, aumentam a capacidade de resiliência diante da doença.

Alguns autores mostram que após passar por um tratamento oncológico, diversos pacientes modificam a maneira de ver e aproveitar a vida, realizam mais os seus desejos e valorizam-se mais, o que os tornam mais capazes diante das dificuldades, além de mais maduros e resilientes (Aaronson et al., 2014; Ristevska-Dimitrovska et al., 2015; Toriy et al., 2013). Tal fato não foi observado no presente estudo, visto que os pacientes tiveram moderado escore de resiliência nos três tempos avaliados, assim como não foi observada diferença entre os tempos.

Quando analisada a depressão, observou-se uma média de 12,67 pontos em T1. No T2, a média foi de 13,81; ambas consideradas depressão leve. Em T3, a média foi de 11,42, que reflete ausência de depressão ou depressão mínima (Gorestein \& Andrade, 1998). Na comparação entre os três tempos, não houve diferença significativa (Tabela 2).

Tabela 2. Média, mediana, intervalo e comparação dos escores totais de depressão nos três tempos avaliados.

\begin{tabular}{ccccc}
\hline & T1 & T2 & T3 \\
Média & $\begin{array}{c}\text { Média } \\
\text { Mediana (Intervalo) }\end{array}$ & $\begin{array}{c}\text { Média } \\
\text { Mediana (Intervalo) }\end{array}$ & Mediana (Intervalo) & \\
\hline \multirow{3}{*}{ Depressão } & 12,67 & 13,81 & 11,42 & \\
& 10 & 12 & 9 & 0,9191 \\
& $(0-45)$ & $(0-40)$ & $(1-40)$ & \\
\hline
\end{tabular}

* p valor $<0,005$ é considerado significante.

Fonte: Autores.

O presente estudo difere de outros que avaliaram a depressão em pacientes oncológicos. A maior parte dos trabalhos mostra que a depressão é uma desordem observada frequentemente em pacientes com CCP e que está ligada as alterações funcionais, tais como na fala, deglutição, respiração, paladar e desfiguração facial no transcurso do tratamento, o que afeta a qualidade de vida (Kim et al., 2016; Rohde et al., 2018; Sawada et al., 2012; Wu et al., 2016).

Pressupõe-se que certos fatores podem ter colaborado para o não desenvolvimento de depressão no grupo estudado. $\mathrm{O}$ primeiro deles pode ser devido à menor ou nenhuma preocupação com sua imagem corporal. Quando interrogados sobre a alteração na aparência após o diagnóstico do CECCP ou com o tratamento, grande parte dos pacientes referiu modificações mínimas ou que não observaram alterações. Tal fato pode justificar a baixa depressão, já que muitos autores que estudaram a depressão em pacientes com CCP, demonstram que as alterações do aspecto físico são importantes preditoras da depressão (Rhoten et al., 2014).

O predomínio do sexo masculino $(89,3 \%)$ na casuística avaliada também pode ter contribuído para a redução da depressão. Estudos que avaliaram a depressão em pacientes oncológicos afirmaram que ela é maior nas mulheres, uma vez que essas têm maior probabilidade de desenvolver desordens depressivas ou relatam mais os sintomas para a equipe e procuram ajuda (Baillargeon et al., 2011; Lee \& Cartmell, 2019).

No CCP, a maior incidência de depressão também é observada em mulheres, o que pode ser explicado pelo fato dessas valorizarem mais a atração facial que os homens. Sendo assim, a desfiguração facial decorrente dos tratamentos é mais impactante para esse grupo e acabam por deixa-las mais vulneráveis para o desenvolvimento da depressão (Katz et al., 2003; Langius et al., 1994).

Sluis et al., (2020) descreveram que mulheres laringectomizadas correm maior risco de impacto negativo da cirurgia que os homens, queixando-se mais de alterações físicas pós operatórias, problemas sexuais, de intimidade e maiores desafios para 
cumprirem as atividades femininas como cuidar da casa, dos familiares e ter boa aparência, reforçando ainda mais o fato de que mulheres com CCP tem maior predisposição as doenças psiquiátricas como a depressão.

Para as análises de correlações, utilizou-se a soma dos escores totais das variáveis nos três tempos. Notou-se correlação negativa e estatisticamente significativa $(\mathrm{rho}=-0,668 ; \mathrm{p}=0,000)$ entre a depressão e a resiliência, mostrando que quanto maior a resiliência, menor a depressão (Gráfico 1).

Gráfico 1: Correlação entre Resiliência e Depressão.

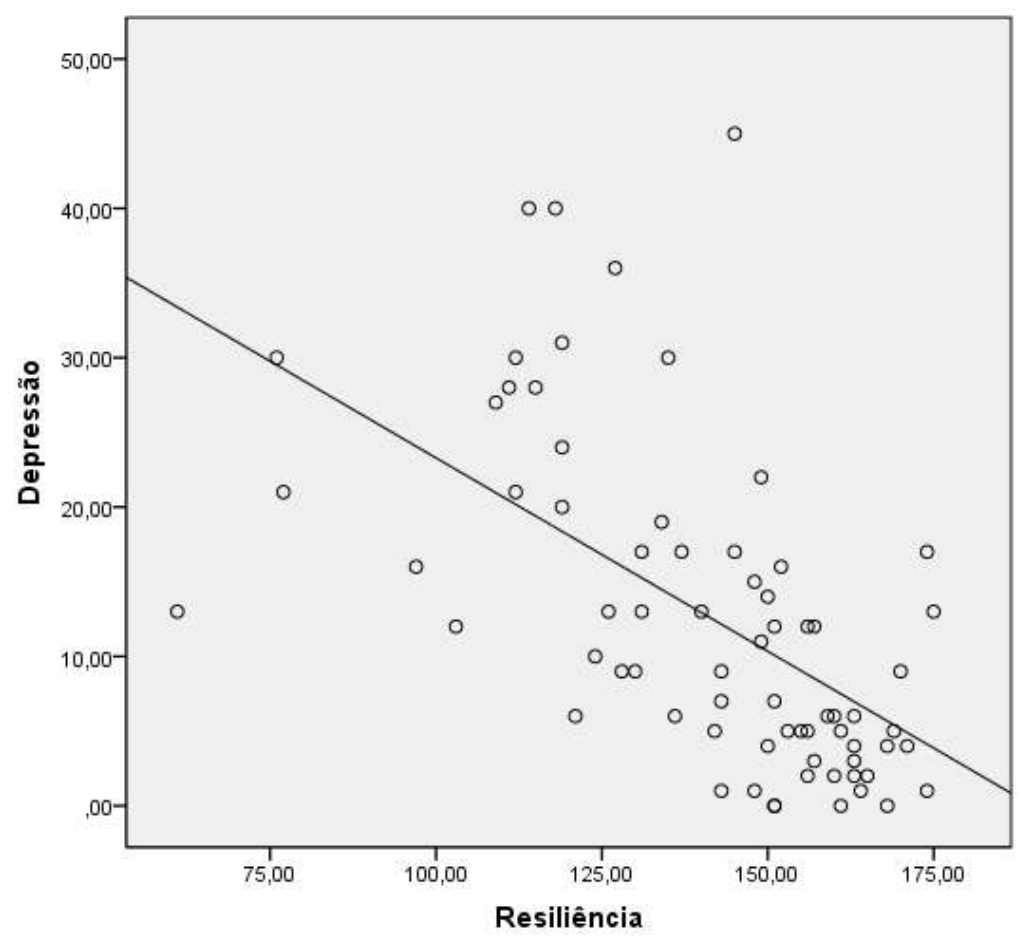

Fonte: Autores.

Escassos estudos avaliaram a correlação entre resiliência e depressão em pacientes com câncer. Sharpley et al., (2014) realizaram essa investigação em indivíduos com tumores malignos na próstata e concluíram relação inversa entre as variáveis, alegando que avaliar a resiliência pode ser de grande valia para prever o desenvolvimento da depressão. Em 2018, os mesmos autores conduziram um estudo com 104 pacientes com câncer de próstata e investigaram o efeito do estresse crônico (mensurado pelos níveis séricos de cortisol) na resiliência e depressão. Demonstrou-se que existe uma correlação inversa entre resiliência e depressão nos pacientes com baixa a moderada concentrações de cortisol (Sharpley et al., 2018).

Estudo realizado com pacientes com câncer de mama avaliou a correlação entre resiliência e depressão e os resultados mostraram que pacientes mais resilientes eram menos deprimidos (Dimitrovska et al., 2015). Achados similares foram descritos em um trabalho conduzido em um hospital chinês com 289 pacientes com câncer de pulmão. As análises de correlações revelaram que a depressão estava associada negativamente à resiliência (Hu et al., 2018), corroborando com os resultados do presente estudo.

O tamanho reduzido da amostra pode ser considerada uma limitação do estudo, uma vez que, somente foram incluídos pacientes com CCECP submetidos a tratamento cirúrgico e com cognitivo preservado. Contudo, compreende um estudo precursor na investigação da relação da resiliência e depressão em pacientes com CCECP submetidos a tratamento cirúrgico. 


\section{Conclusão}

Esse estudo apontou que pacientes com CCECP, submetidos a tratamento cirúrgico, com maior resiliência, apresentam menos sintomas depressivos. Sendo assim, avaliações específicas da depressão e resiliência em pacientes com CCECP se fazem necessárias e podem contribuir para a equipe inferir quais deles podem se beneficiar de uma intervenção precoce, trabalhando estratégias para reduzir os níveis de depressão e aumentar a resiliência, contribuindo para o sucesso do tratamento.

\section{Agradecimentos}

Agradecemos aos pacientes voluntários desse estudo e a toda equipe do Departamento de Cirurgia de Cabeça e Pescoço do hospital onde os dados foram coletados.

\section{Referências}

Aaronson, N. K. et al. (2014). Beyond treatment - Psychosocial and behavioural issues in cancer survivorship research and practice. European Journal of Cancer, Supplement, $12(1), 4-64$.

Aguiar, N., Meira, D. \& Raquel, S. (2011). Resilience en Madres e Jijos con cáncer. Psicooncologia, 8(1), 113-123.

Ahn, M. H. et al. (2015). Suicide in cancer patients within the first year of diagnosis. Psycho-Oncology, 24(5), 601-607.

Algetwi, E., Owens, J. \& Backer, S. R. (2017). Online support groups for head and neck cancer and health-related quality of life. Quality of Life Research. 26(9), 2351-2362.

Baillargeon, J. et al. (2011). Effect of mental disorders on diagnosis, treatment, and survival of older adults with colon cancer. Journal of the American Geriatrics Society, 59(7), 1268-1273.

Bertolucci, P. H. F. et al. (1994). O Mini-Exame do Estado Mental em uma população geral: impacto da escolaridade. Arquivos de Neuro-Psiquiatria, 52(1), $01-07$.

Britton, B. et al. (2012). Baseline depression predicts malnutrition in head and neck cancer patients undergoing radiotherapy. Supportive Care in Cancer, 20(2), $335-342$.

Brucki, S. M. D. et al. (2003). Sugestões para o uso do mini-exame do estado mental no BrasiL. Arquivos de neuro-psiquiatria, 61(3b), 777-781.

Cardoso, G. et al. (2009). Aspectos Psicológicos do Doente Oncológico. Psilogos, 7(1), 8-18.

Clarke, G. et al. (2019). Exploring the relation between patients' resilience and quality of life after treatment for cancer of the head and neck. British Journal of Oral and Maxillofacial Surgery, 57(10), 1044-1048.

Dimitrovska, G. R. et al. (2015). Depression and Resilience in Breast Cancer Patients. Open Access Macedonian Macedonian Journal of Medical Sciences, 3(4), 661-665.

Edmonds, M. F.\& McGuire, D. B. (2007). Treatment Adherence in Head and Neck Cancer Patients Undergoing Radiation Therapy: Challenges for Nursing. Journal of Radiology Nursing, 26(3), 87-92.

Fernández, L. C. \& García, M. L. R. (2012). El poder de la resiliencia generado por el cáncer de mama en mujeres en Puerto Rico1. Revista Puertorriqueña de Psicología, 23, 109-126.

Gomes-Ferraz, C., Rezende, G.\& De Carlo, M. (2019). Uso de tecnologia de comunicação alternativa na avaliação da qualidade de vida de pacientes com câncer de cabeça e pescoço. Cadernos Brasileiros de Terapia Ocupacional, 27(1), 61-71.

Gorestein, C. \& Andrade, L. (1998). Inventário de depressão de Beck : propriedades psicométricas da versão em português. Revista de Psiquiatria Clínica, 25(5), 245-250.

$\mathrm{Hu}$, T. et al.(2018). Relationship between resilience, social support as well as anxiety / depression of lung cancer patients : A cross-sectional observation study. Journal of Cancer Research and Therapeutics, 14(1), $72-77$.

Huang, C. et al. (2019). Development and validation of radiomic signatures of head and neck squamous cell carcinoma molecular features and subtypes. EBioMedicine, 1-11.

INCA, Instituto Nacional de Câncer José Alencar Gomes da Silva. (2019). Estimativa 2020 - Incidência de Câncer no Brasil. [s.l: s.n.].

Katz, M. R. et al. (2003). Psychosocial adjustment in head and neck cancer: The impact of disfigurement, gender and social support. Head and Neck, 25(20, $103-112$.

Kim, S. A. et al. (2016). Pretreatment depression as a prognostic indicator of survival and nutritional status in patients with head and neck cancer. Cancer, 122(1), 131-140. 
Krebber, A. M. H. et al. (2016). Screening for psychological distress in follow-up care to identify head and neck cancer patients with untreated distress. Supportive Care in Cancer, 24(6), 2541-2548.

Langius, A., Bjtirvell, H. \& Lind, M. G. (1994). Functional status and coping in patients with oral and pharyngeal cancer before and after surgery. Head \& Neck, 16 (6), 559-568.

Lee, SJ \& Cartmell, K. (2020). Self - reported depression in cancer survivors versus the general population : a population - based propensity score - matching analysis. Quality of Life Research,29(2), 483-494.

Lima, R. J. DE et al. (2020). Resiliência , capacidade funcional e apoio social de pessoas com sequelas de acidente vascular encefálico. Rev. Eletr. Enferm., 22 (59542), $1-8$, .

Paula, J. M. et al. (2012). Sintomas de depressão nos pacientes com câncer de cabeça e pescoço em tratamento radioterápico : um estudo prospectivo. Rev Latino Am Enfermagem, 20(2), 1-7.

Pesce, R. P. et al. (2005). Adaptação transcultural, confiabilidade e validade da escala de resiliência C. Cad. Saúde Pública, 21(2), 436-448.

Rhoten, B. A. et al. (2014). Body image and depressive symptoms in patients with head and neck cancer: an important relationship. Support Care Cancer, 22(11), 3053-3060.

Rieke, K. et al. (2017). Depression and survival in head and neck cancer patients. Oral Oncology, 65,76-82.

Ristevska-Dimitrovska, G. et al. (2015). Resilience and quality of life in breast cancer patients. Open Access Macedonian Journal of Medical Sciences, 3(4), $727-731$.

Rocha, B. et al. (2017). Características epidemiológicas de pacientes portadores de neoplasias de cabeça e pescoço submetidos à radioterapia em Juiz de Fora MG. HU Revista, 43(1), 71-75.

Rohde, R. L. et al. (2018). Prevalence and sociodemographic factors associated with depression among hospitalized patients with head and neck cancer -Results from a national study. Psycho-Oncology, 27, 2809-2814.

Satin, J. R., Linden, W. \& Phillips, M. J. (2009). Depression as a predictor of disease progression and mortality in cancer patients: A meta-analysis. Cancer, 115(2), 5349-5361.

Sawada, N. O. et al. (2012). Depression, fatigue, and health-related quality of life in head and neck cancer patients : a prospective pilot study. Support Care Cancer, 20, 2705-2711.

Sharpley, C. F. et al. (2018) Limitations in the inverse association between psychological resilience and depression in prostate cancer patients experiencing chronic physiological stress. Psycho-Oncology., 27, 23-228.

Siegel, R. L., Miller, K. D. \& Jemal, A. (2020). Cancer statistics, 2020. CA: A Cancer Journal for Clinicians, 70(1), 7-30.

Sluis, K. E. Van et al. (2020). Women's perspective on life after total laryngectomy : a qualitative study. Int J Lang Commun Disord, 55(2), 188-199.

Sung, H. et al. (2021). Global Cancer Statistics 2020 : GLOBOCAN Estimates of Incidence and Mortality Worldwide for 36 Cancers in 185 Countries. CA Cancer J Clin, 71(3), 209-249.

Talani, C. et al. (2019). Early mortality after diagnosis of cancer of the head and neck - A population-based nationwide study. PLoS ONE, 2, 1-18.

Toriy, A. M. et al. (2013). Percepções, sentimentos e experiências físicoemocionais de mulheres após o câncer de mama. Journal of Human Growth and Development, 23(3), 303-308.

Valadares, Y. D. et al. (2021). Resiliência, depressão e qualidade de vida de pacientes com câncer de cabeça e pescoço no pré operatório. Revista Eletrônica Acervo Saúde, 13(6), 1-10.

Wagnild, G. (2009). A review of the Resilience Scale. Journal of Nursing Measurement, 17(2), 1061-3749.

Wagnild, G. \& Young, H. (1993). Development and psychometric evaluation of the Resilience Scale. J Nurs Meas., 1(2), 165-78.

Wu, Y. S. et al.(2016). Anxiety and depression in patients with head and neck cancer: 6-month follow-up study. Neuropsychiatric Disease and Treatment, 12, $1029-1036$

Zimmaro, L. A. et al. (2018). Depressive symptoms predict head and neck cancer survival: Examining plausible behavioral and biological pathways. Cancer, 124(5), 1053-1060. 